
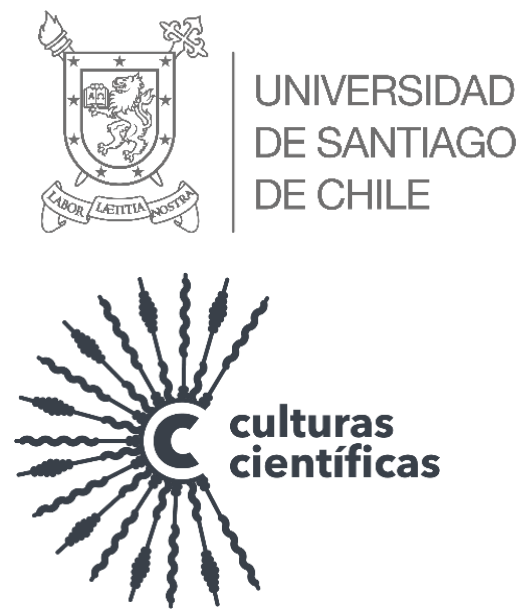

\author{
Miguel Agustín Álvarez Lisboa \\ miguel.alvarez@um.uchile.cl \\ IIF-SADAF-CONICET \\ https://orcid.org/0000-0003-0291-4650
}

Artículo recibido: 11 de mayo de 2021 Artículo aceptado: 9 de junio de 2021 Artículo publicado: 31 de julio de 2021

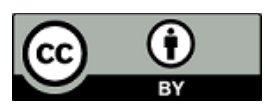

Artículo de Investigación https://doi.org/10.35588/cc.v2i1.4956

\section{Esbozo de una concepción particularista de las Leyes Lógicas}

\author{
Sketch of a Particularist Conception of the Laws of Logic
}

\section{Resumen}

El Anti-Excepcionalismo Lógico afirma que la Lógica es como cualquier otra ciencia. Si esta afirmación es cierta, entonces ella no sólo es revisable, sino que además todo lo que se puede decir sobre las ciencias aplica, mutatis mutandis, para la misma. El propósito de este artículo es explorar esta consecuencia del Anti-Excepcionalismo Lógico, acercando a la Filosofía de la Lógica el marco teórico de las Máquinas Nomológicas de Nancy Cartwright. De acuerdo con esta visión, lo que hay de verdadero en las teorías científicas no está en el mundo sino en sus modelos: sistemas altamente controlados, estables, artificiales y específicos, dentro de los cuales se manifiestan regularidades sistematizables y enseñables. Mi afirmación es que lo mismo puede decirse de la Lógica: ella no captura las Leyes del Pensamiento o de la Razón, porque el pensamiento y la razón no están gobernados necesariamente por leyes. Sobre lo que sí puede decirse que versan las Leyes de la Lógica son metafísicas posibles: historias acerca de qué son las proposiciones, el pensamiento, la verdad y la validez. A fin de ilustrar y defender esta tesis, presento un ejemplo de Máquina Nomológica de la Lógica Clásica, basada en una lectura original del Tractatus de Wittgenstein, e investigo algunas de sus consecuencias. Mi conclusión es que esta imagen de la Lógica impacta en el problema de la revisión de la Lógica Clásica, en la medida en que muestra que dicha revisión no necesariamente responde a un proceso racional de adecuación de la teoría a los hechos porque los "hechos" relevantes no son anteriores a la teoría misma, sino que están moldeados y conducidos por ella.

Palabras clave: Anti-Excepcionalismo Lógico, Abductivismo, Máquinas Nomológicas, Revisión Lógica, Filosofía de la Lógica.

\begin{abstract}
Logical Anti-Exceptionalism claims that Logic is like any other science. If this statement is correct, then Logic is not only revisable, but also everything that can be said about science applies to it, mutatis mutandis. The purpose of this article is to explore this consequence of Logical Anti-Exceptionalism, by approaching the Philosophy of Logic to Nancy Cartwright's theoretical framework of Nomological Machines. According to the latter, the truth of the scientific theories is not in the world, but in its models: highly controlled, stable, artificial, and specific systems, where systematizable and teachable regularities are manifested. I claim that the same can be said about Logic: it does not capture the Laws of Thought or Reason, because thought and reason are not necessarily governed by laws. Laws of Logic are about possible metaphysics: philosophical stories about what propositions, thoughts, truth and validity are. In order to illustrate and defend this thesis, I present an example of Nomological Machine for Classical Logic, based on Wittgenstein's Tractatus, and investigate some of its consequences. I conclude that this image of Logic has an impact on the problem of the revision of Classical Logic, as it shows that this revision does not necessarily respond to a rational process of adaptation of the theory to the facts. This is because the relevant "facts" are not prior to the theory itself but are shaped and driven by it.

Keywords: Logical Anti-Exceptionalism, Abductivism, Nomological Machines, Logical Revision, Philosophy of Logic.
\end{abstract}




\section{Introducción}

¿Es posible revisar la Lógica? Y si es posible, ¿cómo se hace?

El Anti-excepcionalismo Lógico (A-EL) es una tesis filosófica que responde a estas dos preguntas diciendo: la Lógica es una ciencia como cualquier otra. Por lo tanto, es revisable, y esta revisión se hace sobre la base de evidencia empírica, tal como sucede con (las) otras ciencias (Hjortland, 2017; 2019).

Es un hecho conocido que en la actualidad existen varios sistemas lógicos. Ellos gozan de propiedades que, al menos desde un punto de vista matemático, son intrínsecamente interesantes. Pero los filósofos se han preguntado si acaso hay uno, o varios, de estos sistemas que amerite además ser reconocido como el sistema lógico correcto; es decir, aquel que mejor capture cierta noción de validez general o por excelencia $^{1}$.

Existe también relativo acuerdo en que dicha dignidad le pertenece actualmente a la Lógica Clásica: después de todo, la matemática estándar se funda sólidamente en los esquemas de validez que este sistema lógico autoriza y, dada la presencia ubicua de la matemática en nuestras ciencias, parece razonable aceptar que, si hay tal cosa como una lógica correcta, entonces el sistema que mejor da cuenta de ella probablemente sea la Lógica Clásica.

Uno de los debates actuales en Filosofía de la Lógica versa sobre la posibilidad de revisar este estatus privilegiado de la Lógica Clásica. Y, en los últimos años, tanto quienes la defienden como quienes la cuestionan han encontrado en el A-EL un terreno común para su disputa: pues, se piensa, una manera prudente de llevar la discusión es sobre la base de una comprensión científica de lo que significa revisar una teoría.

Sin embargo, es claro que el A-EL dice mucho más que esto; después de todo, la revisión de teorías es sólo uno de los aspectos de la empresa científica y sólo uno de los que interesan a la Filosofía de las Ciencias.

Si el A-EL está en lo correcto, entonces todo lo que las filósofas y los filósofos de las ciencias hayan dicho o puedan decir sobre las ciencias en general aplica, mutatis mutandis s para la Lógica. El propósito de este artículo es explotar esta consecuencia y luego mostrar cómo ella impacta en el debate mismo acerca de la dignidad de la Lógica Clásica, en un punto que es anterior a la revisabilidad. En concreto, lo que haré será aplicar a la Lógica la visión de las teorías científicas expuesta y defendida por Nancy Cartwright en su libro A Dappled world: A study of the boundaries of science (Cartwright, 1999). Esta visión de la Lógica hace implausible una interpretación universalista de la corrección de los sistemas lógicos en general (y de la Lógica Clásica en particular), y da una perspectiva más o menos novedosa de lo que significa la dignidad de la Lógica Clásica y lo que implicaría revisarla.

\footnotetext{
${ }^{1}$ Reconozco que estoy siendo un poco ambiguo; pero esta ambigüedad no es mía. En particular, no es extraño encontrarla en la literatura en torno a la discusión entre pluralistas y monistas (y nihilistas), que es donde más se la usa (véase Russell, 2021). En cualquier caso, mi argumento es compatible con más de una posible forma de comprender esta noción, por lo que no es necesario pronunciarse por una sola acepción.
} 
La estructura del escrito es la siguiente: en la sección 2 presentaré de manera general los elementos que me parecen más sobresalientes de la propuesta de Cartwright y comentaré la imagen de la ciencia que esta última induce; en la sección 3 trasladaré este aparato hacia la Lógica de forma más o menos general y esquemática, para luego, en la sección 4, ahondar en los detalles con un ejemplo concreto. Finalmente, en la sección 5 mostraré de qué forma este análisis influye en la discusión en torno a la dignidad de la Lógica Clásica. Una última sección reúne las conclusiones.

\section{Fábulas y Máquinas Nomológicas}

Siguiendo una larga y venerable tradición, sostengo que las teorías lógicas son acerca de la validez inferencial ${ }^{2}$. Si, como sostiene el A-EL, las teorías lógicas son teorías científicas, entonces ellas deben ser acerca de la validez inferencial en el mismo sentido que las demás teorías científicas son acerca de los fenómenos que ellas estudian.

Pero ¿cómo son las ciencias naturales acerca de la naturaleza? ¿Cómo es, por ejemplo, la Física acerca de los hechos físicos? Según una visión ampliamente generalizada, las teorías científicas refieren al mundo: sus conceptos describen propiedades instanciadas en las cosas y sus leyes capturan regularidades que se dan "allá afuera" de manera efectiva y por doquier.

Tomemos por ejemplo la ecuación de aceleración en un Movimiento Circular Uniforme (MCU). Donde $\omega$ es la velocidad angular, $r$ el radio de la circunferencia recorrida y $a$ la aceleración del cuerpo en movimiento, la ley:

$$
a=-\omega^{2} r
$$

describe una igualdad a la que responde la totalidad de fenómenos físicos que corresponden a este tipo de movimiento.

El libro A Dappled world: A study of the boundaries of science (Cartwright, 1999) ofrece una comprensión alternativa de lo que son las teorías científicas y sus leyes. Para la autora, las leyes científicas no son verdades acerca de la naturaleza: en tanto universales, ellas son falsas; y cuando puede decirse que son verdaderas, ellas lo son sólo en ambientes particulares y controlados. Son leyes ceteris paribus: generalizaciones relativizadas a casos en los que se cumplan una serie de condiciones favorables.

Las leyes ceteris paribus no le son enteramente ajenas a la visión tradicional ${ }^{3}$ : las encontramos, por ejemplo, en ciencias como la Biología o la Economía. Pero, por regla general, se les trata como ciudadanos

\footnotetext{
${ }^{2}$ Tan larga que podría rastrearse a, por lo menos, Pedro Abelardo (King y Arlig, 2018), y tan venerable como para haber sido sostenida por lógicos de la talla de Alfred Tarski, Michael Dummett o John Etchemendy; y más recientemente por Sara Negri, Greg Restall, J. C. Beall y varios otros (Beall, Restall y Sagi, 2019). Se trata, por lo demás, de una posición bastante estándar en la discusión sobre A-EL.

${ }^{3}$ Estoy llamando aquí "visión tradicional" a lo que Cartwright llama en su libro la visión fundamentalista (fundamentalist) de las leyes científicas y no a lo que comúnmente se conoce como "visión heredada" en Filosofía de las Ciencias, si bien ambos conceptos pueden tener elementos en común.
} 
de segunda categoría. Por apelar a una conocida metáfora de Popper (1965), la visión tradicional asume que, en el fondo, "todas las nubes son relojes", y una ley ceteris paribus es un recurso imperfecto, faute de mieux; las auténticas leyes científicas deberían ser determinísticas, necesarias y universales, como las de la Física. Cartwright se alinea, en cambio, de lado de quienes afirman que "todos los relojes son nubes"; y que las leyes de la Física son en realidad como las de la Economía o la Biología, sólo que sus cláusulas ceteris paribus no siempre están a la vista. Sin ánimo de comprometerse muy fuertemente con esta imagen, la autora dice:

Pienso que los objetos naturales son como las personas en sociedades. Su conducta está constreñida por algunas leyes específicas y por un puñado de principios generales, pero no está determinada en detalle, ni siquiera estadísticamente. Lo que ocurre, en la mayoría de los casos, no está dictado por leyes en lo absoluto (Cartwright, 1980, p.161)4.

Cartwright ilustra la relación entre el modelo y la ley científica con la relación entre la fábula y su moraleja. "Las fábulas transforman lo abstracto en concreto, y al hacerlo, sostengo, funcionan como modelos en física. [...] la relación entre la moraleja y la fábula es como la que hay entre la ley científica y un modelo" (p. 37). La fábula reviste ${ }^{5}$ a su moraleja, explotando intuiciones y conocimientos de sentido común acerca de ciertos animales a fin de ilustrar un punto.

Tomemos por ejemplo la siguiente fábula (Barros Grez, 1888, Fábula 82ª (A), p. 124)

- ¿Por qué goza de clara nombradía

la abeja, y para mí sólo hay ultraje?

"Soy su igual, pues, que soy de su linaje"

Lleno de orgullo, un zángano decía.

Replicóle una hormiga, que le oía:

- ¿¿Quiere usted valer algo? Pues trabaje;

Porque, si ha de vivir ocioso,

No será más que un Zángano orgulloso."

Esta fábula presenta un diálogo entre dos animales: el zángano y la hormiga. Ellos son, respectivamente, el ocioso y la trabajadora. Pero estos animales no representan estos conceptos (como lo haría una metáfora); más bien, ellos los instancian, como particulares de un universal. La hormiga y la abeja no son elegidas porque simbolicen trabajadoras esforzadas, sino porque, de hecho, lo son; y el zángano, en comparación con la abeja, porque no trabaja. Dicho de otra forma: los conceptos abstractos

${ }^{4}$ Esta y todas las demás traducciones desde el inglés son mías.

${ }^{5}$ La palabra alemana que usa G. E. Lessing, a quien Cartwright sigue en estos pasajes, es: Einkleiden, que ella traduce al inglés como fit out. Yo estoy traduciendo del inglés antes que del alemán, pues me interesa el sentido que a Cartwright le interesa.

${ }^{6}$ He modificado la ortografía del original. 
de "laboriosidad" y "ociosidad" se revisten de los rasgos concretos que exhiben hormigas, abejas y zánganos, con el fin de hacer concreta la moraleja.

Esta misma relación es la que existe, para Cartwright, entre los conceptos abstractos que las leyes científicas ponen en relación y los modelos que fabricamos para ellos. Dice Cartwright:

Usamos animales como el faisán o el lobo [en nuestro caso, la abeja y la hormiga] porque sus caracteres nos son muy conocidos. Sólo necesitamos decir sus nombres para emular qué características en general tienen ellos- jactancia, debilidad, testarudez, orgullo, [laboriosidad, ociosidad] o similares. En Física es más difícil. En general no es bien sabido cuáles son las situaciones estereotípicas en las que se exhiben varias de las formas funcionales de la fuerza. Esto es lo que el físico profesional debe averiguar, y lo que el aspirante a físico debe aprender ${ }^{7}$ (Cartwright, 1999, p.43).

Según esta idea, las leyes físicas son acerca de los fenómenos físicos de la misma forma como la moraleja es acerca del carácter de ciertas personas: expresan verdades imprecisas y amplias, enunciadas a través de conceptos abstractos que pueden instanciarse en situaciones concretas. La tarea de la científica y el científico es posibilitar situaciones en las cuales dicha instanciación pueda ocurrir de manera prístina, sistematizable y enseñable: y tal objetivo se alcanza con la fabricación de una máquina nomológica (MN).

En pocas palabras, una MN es "un arreglo (suficientemente) fijo de componentes, o factores, con capacidades (suficientemente) estables que, en el tipo correcto de ambiente (suficientemente) estable dará lugar, de manera repetible, a las diversas conductas regulares que representamos mediante nuestras leyes científicas" (Cartwright, 1999) ${ }^{8}$. Para Cartwright, la naturaleza no está organizada de acuerdo con leyes determinísticas y universales, sino que sus hechos y objetos exhiben capacidades; éstas, en diferentes tiempos y contextos, pueden dar lugar a diversos y a veces impredecibles resultados. Se acercan más al uso corriente de palabras como "laborioso" u "ocioso", que no describen características bien definidas y estables de una persona, sino más bien abren un espectro difuso de posibilidades de realización.

Volvamos a la ecuación (1). En tanto proposición, esta ley científica es falsa respecto de la gran mayoría, sino de todos, los fenómenos de MCU en el universo: el valor de $a$, dados $\omega$ y $r$ sencillamente nunca se da, o se da sólo algunas veces, pues en los sistemas reales hay muchos factores en juego que esta ecuación ignora. Por ejemplo, el roce del medio en que sucede el movimiento. Según la visión tradicional, este hecho se explica diciendo que dichas influencias pueden ser también descritas mediante ecuaciones legaliformes, y que en última instancia el fenómeno real es descrito y predicho totalmente por la composición ideal de todas las leyes relevantes. Esta pretensión está injustificada, según Cartwright, o a lo sumo impone una regularidad en la naturaleza que no está fundada en otra cosa que una esperanza metafísica: que "todas las nubes sean relojes".

${ }^{7}$ La palabra 'grouse', que vertí en 'faisán', significa más propiamente urogallo; pero preferí usar un nombre más universalmente conocido en habla hispana, para conservar el sentido de lo que Cartwright (y Lessing) quiere decir.

${ }^{8}$ El énfasis es mío. 
Pero, si (1) es falsa respecto de la totalidad de los fenómenos de MCU, ¿respecto de qué ella es verdadera? Pues sólo de sistemas muy específicos y altamente controlados. En este sentido es una ley ceteris paribus, pues sólo si

1. nada interfiere en el sistema,

2. los únicos objetos y fuerzas medibles son los relevantes, y

3. toda posible influencia dentro del mismo sistema es también una fuerza,

se cumple la igualdad que ella pregona. Tomemos por caso un cuerpo $A$, atado mediante una cuerda de material $M$ a un punto fijo $X$, girando libremente alrededor de él. La ecuación (1) se verifica sobre este sistema si y sólo si

1. nada interfiere con el movimiento de $A, M$ es resistente e inelástico, $X$ está firme e inmóvil...

2. no hay fuerza de gravedad, ni roce con el medio, ni fricción entre la cuerda y $X$ o $A \ldots$

3. a menos que dichas influencias puedan cuantificarse y considerarse como variables independientes en el sistema.

En este caso no es difícil obtener instancias apropiadas para $A, M$ y $X$. Por ejemplo, podríamos atar una mancuerna con una cuerda de saltar y pedirle a una deportista lo suficientemente fuerte que la haga girar manteniendo su brazo estable en su lugar. Si el peso de la mancuerna no es ni mucho ni muy poco, la cuerda está bien atada y la deportista es lo suficientemente fuerte y tiene un buen dominio de su cuerpo, el sistema se comportará como esperamos y una medición de los valores relevantes debería dar, con muy bajo margen de error, algo cercano a lo mandado en (1).

El sistema descrito recién es una MN: un caso paradigmático, un ejemplar simple y transparente que permite a los componentes exhibir sus capacidades (mecánicas, en este caso) de una forma particularmente clara e ilustrativa. Y las leyes científicas son afirmaciones acerca de estos sistemas, y no enunciados universales que los tienen a ellos como casos particulares. La MN

(...) tiene componentes fijos, con capacidades conocidas dispuestas en una configuración estable. (...) La afirmación que yo hago es que siempre necesitamos una máquina como ésta para obtener las leyes - cualquier tipo de leyes, causales o lo que fuera. A veces Dios provee los dispositivos - como en el caso de los sistemas planetarios - pero la mayoría de las veces debemos proporcionárnoslos nosotros mismos, en cortes e iglesias, instituciones y fábricas. (Cartwright, 1999, p.122)

Es importante notar que Cartwright no está postulando entidades extrañas en la empresa científica; más bien, está mirando los mismos elementos que ya conocíamos bajo un prisma distinto. Las MN son "un concepto filosófico, como 'ley incondicional' o 'teoría completa' o 'determinismo universal', una manera de categorizar y comprender qué es lo que sucede en el mundo" (Cartwright, 1999, p. 57). Lo que propone la autora con este concepto es una inversión de la relación usualmente asumida entre las leyes científicas y los experimentos paradigmáticos: en lugar de tener muy buenos ejemplos de regularidades que se dan por doquier en el mundo, lo que hay son máquinas ingeniosamente diseñadas para explotar ciertas capacidades de forma regular, estable y predecible. 


\section{Máquinas Nomo-Lógicas}

Si las teorías lógicas son teorías científicas, entonces sus leyes son a sus modelos como las moralejas a las fábulas. Y los conceptos con los que dichas leyes están formuladas no refieren a propiedades objetivas de argumentos o inferencias, sino a capacidades de estos últimos. Y estas capacidades son exhibidas y sistematizadas por MN particulares, a las que llamaré, a falta de un mejor nombre, Máquinas Nomo-Lógicas (MN-L) 9 .

Comencemos por hacer un breve recuento de las características principales de la MN en general. Dijimos en la sección anterior que una MN es

1. un caso particular y artificial (en la mayoría de los casos), altamente controlado y estable, dotado de una narrativa que permite

2. explotar las capacidades de sus componentes para producir regularidades legaliformes.

Una MN-L será esencialmente lo mismo, aunque con algunas diferencias significativas. En concreto, veo a una MN-L como un cuentito en el que figuran conceptos e ideas filosóficas. Si las MN de las ciencias empíricas son escenarios experimentales, entonces una MN-L es un experimento mental (Gedankenexperiment): una narrativa que "bombea ciertas intuiciones" (Dennett, 1995; 2013) en una dirección predecible. Dichas intuiciones son las capacidades del lenguaje natural que la Lógica explota para producir sus regularidades.

Sobre el componente narrativo y la artificialidad de las MN-L me referiré más en profundidad en la sección siguiente. En esta aclararé qué estoy entendiendo por "intuiciones" y en qué sentido digo que se corresponden con las capacidades de Cartwright.

La literatura lógica contemporánea atribuye un lugar relativamente destacado a la noción de "intuición lógica" (cf. Hjortland, 2019). Se dice, en efecto, que la base de evidencia sobre la que se erige la Lógica está conformada de manera significativa, si es que no crucial, por estas intuiciones acerca de cuándo, o qué, argumento es válido o inválido. Sin embargo, mi posición en este punto es más bien cauta. Sigo aquí a Tajer (2020), para quien, aunque algunas de nuestras intuiciones lógicas sí emergen de ciertos usos cotidianos (como aquellas acerca de las conectivas lógicas), otras (como aquellas acerca del concepto de Validez) tienen "un carácter teórico inescapable" (Tajer, 2020, p.244). Aunque, para mí, esta conclusión es una consecuencia de asumir un A-EL ${ }^{10}$ : y el caso de la Lógica es sencillamente el mismo que para otras ciencias, donde algunos conceptos tienen correlatos más o menos inmediatos fuera de la práctica científica, pero otros no. Por ejemplo, el concepto de energía suele disparar intuiciones muy distintas en personas con o sin educación científica. En particular, existe cierto uso secular de la palabra energía de connotaciones espirituales y místicas antes que mecánicas; estos usos ni se condicen ni son compatibles con lo que la Física entiende por esta palabra. De manera similar, una persona no educada en Lógica difícilmente aceptará como "intuitivo" un razonamiento que pase de premisas contradictorias a una

\footnotetext{
${ }^{9}$ Una manera sensata de pronunciar en voz alta este nombre sería: "Máquina Nomológica de la Lógica" y no, ciertamente, "Máquina Nomo-guión-Lógica".

${ }^{10}$ Tajer comenta que su posición es compatible con el A-EL, pero su argumentación no lo presupone.
} 
conclusión arbitraria, incluso cuando podría asentir a la afirmación de que validez es preservación de verdad, o reconocer exitosamente otros casos como válidos. Tajer, al comentar cierta vertiente del inferencialismo que se pretende fundado en intuiciones pre-teóricas, dice:

Las reglas adicionales como Doble Negación o ex falso sequitur quodlibet prueban que la lógica no puede reducirse a las reglas que supuestamente constituyen el significado de los conectivos. Como bien sabemos, las reglas de introducción y eliminación de los conectivos clásicos sólo nos permiten obtener lógica minimal. En particular, no hay ninguna regla que nos permita inferir cualquier cosa a partir de una contradicción. Sin embargo, aceptamos esa regla siempre que tengamos el concepto teórico de validez y nos demos cuenta que la inferencia ex falso sequitur quodlibet preserva verdad. (Tajer, 2020, p. 249)

Conceptos como los anteriores - energía en el caso de la Física; Validez en el caso de la Lógica — son abstracciones teóricas que el modelo debe instanciar y explicitar con casos y aplicaciones concretos. Para poder llegar a dichos particulares, la teoría se sirve de otros conceptos que, aunque también abstractos, están más cerca de la comprensión y los usos comunes. Esto es a lo que se refería Cartwright en la cita de la sección 2, cuando luego de explicar la función que cumplen los animales en la fábula dice:

En general no es bien sabido cuáles son las situaciones estereotípicas en las que se exhiben varias de las formas funcionales de la fuerza. Esto es lo que el físico profesional debe averiguar, y lo que el aspirante a físico debe aprender.

Por lo tanto, no todas las intuiciones relevantes para la lógica son pre-teóricas; pero, independiente de su anterioridad a la teorización, lo relevante es que en todo caso son ellas lo que la MN-L explota. Este punto se verá más claramente si volvemos la atención a aquellas intuiciones que sí puede decirse que provienen del uso profano. Como dice Tajer, a menudo nuestras investigaciones lógicas están guiadas por intuiciones pre-teóricas acerca de las conectivas lógicas. Un caso claro de esto es el de la negación. Hay varias lógicas alternativas que se distinguen por la forma en que manejan la negación; todas ellas, por apelaciones más o menos explícitas a usos intuitivos o prácticas comunes. Es por esta razón que, en Sylvan (1999), la negación es caracterizada como un determinable susceptible de tener distintos determinantes:

La distinción entre determinable y determinante proviene del diccionario. Determinable es aquello que es 'capaz de ser determinado; propio a ser determinado', y los determinantes son aquello que está propiamente determinado, la forma específica. De acuerdo con la teoría miniatura [miniature] de las partículas lógicas, muchas nociones lógicas clave —la mayoría de las partículas lógicas principales - son determinables en carácter, con tipos determinantes más específicos subsumidos bajo un determinable que los cubre, de manera similar a como varios colores determinados caen bajo el determinable color. La negación es uno de estos objetos, con una función determinable cubriente, una función que es, naturalmente (y, hasta ahora, trivialmente), aquella de negar de alguna manera. [...] Entre los varios determinantes sentenciales, por ejemplo, encontramos las negaciones conexiva, clásica, intuicionística, ortológica, relevante y todavía otras. (Sylvan, 1999, p.300) 
Esta misma distinción entre determinables y determinantes es la que usa Cartwright al explicar la diferencia entre disposiciones y capacidades (Cartwright, 1999, p. 64) ${ }^{11}$. Por lo tanto, me parece que estos tres autores (Cartwright, Sylvan y Tajer) convergen en la misma idea: lo que Sylvan llama "la negación como determinable", y que sería lo que Tajer entiende por intuición pre-teórica de negación, se corresponde con una capacidad (en el sentido de Cartwright) que tienen ciertos usos del lenguaje natural. Esta capacidad es la que la MN-L explota para dar origen a "la negación como determinante", es decir, la operación de negación dentro de una teoría lógica específica.

En concreto, cuando digo que las intuiciones pre-teóricas son capacidades del lenguaje natural, quiero decir que ciertas construcciones y usos de este último pueden servir como lo espera la Lógica. Por ejemplo, el adverbio "no" es capaz de comportarse como la negación verifuncional de la Lógica Clásica, o como la función arrow falsum del intuicionismo, o incluso como la operación de anulación de las lógicas conexivas. Dicho de otro modo, las Leyes de la Lógica no enuncian verdades acerca del "uso correcto" o "perfecto" de ciertas palabras del lenguaje natural, sino que se sirven de las posibilidades abiertas de estas palabras para dar lugar a ese uso nuevo, sistemático y prolífico, que es el discurso deductivo. Ahondaré un poco más en esta idea luego de presentar el ejemplo concreto de la siguiente sección.

\section{Un ejemplo de Máquina Nomo-Lógica}

En esta sección voy a describir una MN-L para la Lógica Clásica. Aunque tal vez es más apropiado decir que voy a identificar dicha MN-L en un texto que ya la describe detalladamente: el Tractatus Logico-Philosophicus (TLP) de Ludwig Wittgenstein (2018).

Antes de decir algo más, me parece necesario hacer el siguiente alcance: es sabido que la obra de Wittgenstein, y en particular el TLP, se presta para (y ha recibido) muy variadas interpretaciones en el último siglo (como dijera Jean-Yves Girard, Wittgenstein es como Nostradamus: en su obra se puede encontrar todo, pero siempre en retrospectiva). No obstante, no soy excesivamente optimista en este punto. En particular, no pretendo decir que Wittgenstein haya querido presentar en el TLP algo como lo que yo estoy entendiendo por MN-L. Muy por el contrario, Wittgenstein difícilmente podría considerarse un anti-excepcionalista: ni su visión de la lógica ni su visión de la ciencia hacen posible una lectura así (véase, por de pronto, la proposición 6.13) ${ }^{12}$. Por lo que cualquier lectura del texto que pretenda hacer un mínimo de justicia a las intenciones de su autor debería alejarse de esta interpretación. Mi lectura está mucho más centrada en la literalidad del texto que en su contexto e intención de producción.

He dicho en la sección anterior que una MN-L es un cuentito filosófico que bombea intuiciones (preteóricas y teóricas) a fin de producir ciertas regularidades. En el caso del TLP, el cuentito tiene por protagonista a la Lógica, un entramado de formas comunes que atraviesa al mundo, al pensamiento y al

${ }^{11}$ Ella, a su vez, rastrea la idea hasta The Concept of Mind de Gilbert Ryle.

${ }^{12}$ De aquí en adelante citaré las proposiciones del TLP con la numeración que tienen en el texto, anteponiendo simplemente un "pr." (proposición) y prescindiendo del número de página de la edición de referencia (Wittgenstein, 2018). En ocasiones marcaré un número con un guion, queriendo indicar con esto que la referencia es a esa proposición y a todas sus proposiciones subordinadas (por ejemplo, 6.23- se refiere a las proposiciones 6.23, 6.231, 6.232, 6.2321, 6.2322, 6.2323, 6.233, 6.2331, 6.234 y 6.2341). 
lenguaje y hace posible la figuración. Cada uno de estos tres dominios es inefable: se muestra, pero no puede ser descrito lingüísticamente sino por un uso extralimitado del lenguaje (que sería, según lo sugiere pr. 6.54, el que se usa en el TLP mismo). Por un lado, tenemos el mundo, constituido por la totalidad de los hechos del espacio lógico (prs. 1.13, 2.0121, 2.013) que se dan efectivamente (prs. 1, 1.1). La sustancia de estos hechos, y por consiguiente del mundo, son los objetos. Ellos tienen propiedades internas y externas, siendo las primeras lo que determina el total de posibilidades de las segundas (prs. 2.01-): y el darse efectivo de la propiedad externa de uno o más objetos es un hecho, es decir, un estado de cosas (prs. $2.0141,2.0272$ ). Estos estados de cosas, efectivos o posibles son los hechos (pr. 2), y es lo que el sujeto representante (que es trascendental: pr. 5.631) se figura en la proposición. El pensamiento, en ese sentido, es la proposición que representa un hecho posible del mundo (pr. 4), y que se deja expresar por medio del lenguaje (pr. 4.001). Las proposiciones son entonces verdaderas o falsas en virtud de los hechos que ellas representan, y su posibilidad de ser verdaderas o falsas está determinada por la forma en que los hechos del espacio lógico se dan o no se dan.

El itinerario completo que da cuenta del hecho filosófico de que las proposiciones sean verdaderas o falsas recorre, al menos, las siguientes proposiciones del texto:

1. "...la totalidad de los hechos determina lo que es el caso y también todo cuanto no es el caso" (pr. 1.12).

2. "El darse y no darse efectivos de estados de cosas es la realidad. (Llamamos hecho positivo al darse efectivo de estados de cosas; al no darse efectivo, hecho negativo)" (pr. 2.06).

3. "La figura representa su objeto desde fuera (su punto de vista es su forma de representación); por ello representa su objeto correcta o falsamente" (pr. 2.173).

4. "La figura concuerda o no con la realidad; es correcta o incorrecta, verdadera o falsa" (pr. 2.21).

5. "Su verdad o falsedad [de la figura] consiste en el acuerdo o desacuerdo de su sentido con la realidad" (pr. 2.222).

6. "La realidad tiene que quedar fijada por la proposición en orden al sí o al no. Para ello ha de ser enteramente descrita por la misma. La proposición es la descripción de un estado de cosas" (pr. 4.023).

7. "Solo en la medida en que es una figura de la realidad la proposición puede ser verdadera o falsa" (pr. 4.06).

8. "La proposición representa el darse y no darse efectivos de los estados de cosas" (pr. 4.1).

9. "El sentido de la proposición es su coincidencia y no coincidencia con las posibilidades del darse y no darse efectivos de los estados de cosas" (pr. 4.2).

10. "La proposición más sencilla, la proposición elemental, afirma el darse efectivo de un estado de cosas" (pr. 4.21).

11. "Si la proposición elemental es verdadera, el estado de cosas se da efectivamente; si la proposición elemental es falsa, el estado de cosas no se da efectivamente" (pr. 4.25).

12. "La proposición es la expresión de la coincidencia y no coincidencia con las posibilidades veritativas de las proposiciones elementales" (pr. 4.41). 
13. "Las posibilidades veritativas de las proposiciones elementales son las condiciones de la verdad y falsedad de las proposiciones" (pr. 4.41).

14. "La expresión de la coincidencia y no coincidencia con las posibilidades veritativas de las proposiciones elementales expresa las condiciones veritativas de la proposición. La proposición es la expresión de sus condiciones veritativas (pr. 4.431).

La narración que cuentan estas proposiciones (y sus aledañas) justifica el sistema lógico que presenta en el grueso del ensayo (prs. 4.24 a 6.03), y que se corresponde con la Lógica Clásica. Las reflexiones que hace a continuación se fundan, a su vez, en el hecho de que el sistema al que se refieren no es más que una aplicación sistemática de la historia que ha contado en la primera parte del escrito. Por ejemplo, cuando dice en 6.1: "las proposiciones de la lógica son tautologías", o más adelante, en 6.113: "Que a la sola luz del símbolo pueda reconocerse que son verdaderas es característica peculiar de las proposiciones lógicas, y este hecho encierra en sí toda la filosofía de la lógica". Estas observaciones no tienen sentido fuera del entramado conceptual que da cuenta de lo que son las tautologías (prs. 4.46-), lo que significa comprender una proposición (pr. 4.024), la distinción entre decir y mostrar (prs. 4.12-) o la relación entre mundo y lenguaje (prs. 5.6-).

El TLP provee una base filosófica para comprender por qué algunas de las cosas que la Lógica Clásica dice que son necesarias (es decir, sus leyes) lo son efectivamente. Por ejemplo, una de estas leyes dice que no hay contradicciones verdaderas. Si entendemos "contradicción" como la mutua afirmación de una proposición y su negación ${ }^{13}$, entonces podemos ir al TLP para hallar la razón: en la medida en que una proposición expresa el darse o no darse de un estado de cosas, podemos representar este darse y no darse por los símbolos V y F; luego, podemos representar la totalidad de las combinaciones posibles entre una proposición y su negación con la tabla ${ }^{14}$ :

\begin{tabular}{|c|c|c|}
\hline$p$ & $\sim p$ & $p \& \sim p$ \\
\hline $\mathrm{V}$ & $\mathrm{F}$ & $\mathrm{F}$ \\
\hline $\mathrm{F}$ & $\mathrm{V}$ & $\mathrm{F}$ \\
\hline
\end{tabular}

La que nos muestra que no hay posibles estados de cosas en los que la contradicción sea verdadera. Esta explicación sólo es satisfactoria si aceptamos todo lo que previamente se ha establecido en el TLP como lo fundante de esta explicación: sus doctrinas sobre el mundo, la proposición, la verdad, la falsedad, el pensamiento, la posibilidad de su representación, la distinción entre decir y mostrar, etc.

Expuesto de esta forma, es fácil notar que el TLP cumple con los requisitos que dimos en la sección anterior para ser una $\mathrm{MN}$, con la gran diferencia de que sus cláusulas ceteris paribus no son físicas sino metafísicas. Esto es lo que distingue, en mi opinión, a una MN de una MN-L.

\footnotetext{
${ }^{13}$ Otra forma truística — y por ende menos interesante— de entender la palabra "contradicción" sería apelar a su uso literal en el TLP como "proposición idénticamente falsa".

${ }^{14}$ Uso la notación de Wittgenstein sólo en este ejemplo.
} 
Pero al afirmar que el TLP es una MN-L estoy diciendo algo todavía un poco más fuerte: que sin su aparataje —o alguno análogo - las leyes de la Lógica Clásica no se pueden justificar, pues ni siquiera son verdaderas. En efecto, para poder dar cuenta de cualquier ley lógica, como la de No Contradicción ("no hay contradicciones verdaderas") u otra cualquiera, siempre será necesario excluir cuidadosamente cierto tipo de situaciones y usos del lenguaje natural que podrían servir de contraejemplos. Sin estas constricciones obvias es posible fabricar, con relativa facilidad, ejemplos quizás inverosímiles, pero siempre estrictamente correctos ${ }^{15}$. Son lo que Cartwright llama condiciones de blindaje (shielding conditions), y que en la Lógica se corresponden con todos aquellos caveat para "formalizar correctamente" que transmitimos a nuestros estudiantes en su primer curso de Lógica. Es aquel proceso en el cual predicados vagos, paradojas de autorreferencia, descripciones definidas, indexicales y toda otra batería de construcciones lingüísticas gramaticalmente aceptables, con usos naturales perfectamente legítimos e incluso interés filosófico intrínseco, son dejados de lado para permitir que los casos paradigmáticos de validez puedan aparecer con claridad. En forma perfectamente análoga, por lo demás, a cuando las profesoras de Física ejemplifican sus leyes en sistemas ideales, con cuerpos de masa despreciable, sin roce y sin interferencias.

Hoy en día pocas personas dedicadas a la filosofía o a la Lógica aceptarían sin reservas las doctrinas expuestas en el TLP, aun cuando estén dispuestas a aceptar la Lógica Clásica como correcta. Sin embargo, las MN no son únicas para cada teoría, y lo mismo ocurre con las MN-L. Es posible contar un cuentito análogo al de Wittgenstein, pero prescindiendo de sus doctrinas metafísicas más arriesgadas. Aunque mi impresión es que todo defensor de la Lógica Clásica acepta, implícita o explícitamente, ciertas doctrinas metafísicas que, por cumplir la función del TLP, son, en alguna medida, isomórficas a las que se presentan en él. Una propuesta reciente que podría servir para ejemplificar este punto es la de Williamson. En escritos recientes (Williamson, 2013; 2014) el filósofo ha defendido un deflacionismo de la noción de Validez, de acuerdo con el cual las leyes lógicas son simplemente enunciados verdaderos de máxima generalidad (formulados en un lenguaje de predicados de segundo orden). Por ejemplo, la ley de No Contradicción dice simplemente que ninguna propiedad se instancia a la vez que su negación en algún individuo, es decir:

$$
\forall X \forall x \neg(X x \wedge \neg X \mathrm{x})
$$

Esta idea es reminiscente de la tesis tractatariana según la cual "las proposiciones de la lógica son tautologías" (pr. 6.1; véanse, además, prs. 6.1224, 6.1231, 6.124); y aun cuando pueda decirse que la propuesta de Williamson no es sólo un reciclaje de la idea de Wittgenstein, es claro que depende de las mismas condiciones de blindaje (shielding conditions): en este caso, todas las que hacen que un contraejemplo para el universal de segundo orden en (3) sea efectivamente imposible ${ }^{16}$.

${ }^{15}$ Véanse, por ejemplo, los ejemplos de contradicciones verdaderas presentados en da Costa (1980) y Priest (2006).

${ }^{16}$ Para ser justos con Williamson, él no cree que la posibilidad de encontrar contraejemplos a las leyes lógicas sea necesariamente una razón para abandonarlas. Su abductivismo y su defensa de la Lógica Clásica son, por tanto, menos sensibles a la posibilidad metafísica de contradicciones verdaderas, o a las paradojas semánticas, que lo que cabría esperarse en primera 
En la sección 2 destacamos que un aspecto importante de las MN para Cartwright es su artificialidad: ellas no se dan naturalmente en el mundo (salvo contadas excepciones), y somos nosotros los que tenemos que fabricarlas. Por supuesto, el caso de las MN-L tiene que ser ligeramente distinto, por la naturaleza misma de las máquinas involucradas; pero creo que ellas en lo esencial permanecen igualmente artificiales, en la medida en que descansan sobre presuposiciones metafísicas ideales. Con esto último quiero decir que ellas no necesariamente son presentadas como el sistema último del mundo; es decir, no son ontologías postuladas sino sólo postulables. Presumiblemente, Wittgenstein en el TLP no afirmó que "el mundo es todo lo que es el caso" como mera hipótesis metodológica, sino que él efectivamente creía (en la medida en que esto tenga sentido) que el mundo es todo lo que es el caso; pero, en tanto MN-L, basta con que la ontología del TLP sea internamente consistente, un "modo razonable" de dar cuenta del mundo (y del razonamiento).

Por lo demás, Wittgenstein en el TLP ya manifiesta una temprana comprensión de este punto. Por ejemplo, cuando dice: "Ahora queda claro por qué se ha sentido a menudo como si las "verdades lógicas" pudieran ser postuladas por nosotros: podemos, en efecto, postularlas en la medida en que podemos postular una notación satisfactoria" (pr. 6.1223), y un poco más adelante, en la pr. 6.124: "Las proposiciones lógicas describen el armazón del mundo o, más bien, lo representan. No "tratan" de nada. Presuponen que los nombres tienen significado, y las proposiciones elementales, sentido; y ésta es su conexión con el mundo [el énfasis es mío]”. Y en Investigaciones Filosóficas su conciencia de este punto llegará a hacerse todavía más madura:

F. P. Ramsey insistió una vez conversando conmigo en que la lógica es una 'ciencia normativa'. [...] En filosofía comparamos frecuentemente el uso de una palabra con juegos y cálculos de reglas fijas, pero no podemos decir que quien usa el lenguaje tenga que jugar tal juego. - Pero si se dice entonces que nuestra expresión verbal se aproxima sólo a esos cálculos, se está con ello al borde de un malentendido. Pues entonces puede parecer como si hablásemos en lógica de un lenguaje ideal. Como si nuestra lógica fuera una lógica, por así decirlo, para el vacío. - Mientras que la lógica no trata del lenguaje —o del pensamientoen el sentido en que una ciencia natural trata de un fenómeno natural, y lo más que puede decirse es que construimos lenguajes ideales. Pero aquí la palabra "ideal" sería desorientadora, pues suena como si esos lenguajes fuesen mejores, más perfectos, que nuestro lenguaje corriente; y como si le tocase al lógico mostrarles finalmente a los hombres qué aspecto tiene una proposición correcta [este énfasis es mío] (Wittgenstein, 2014, par. $81)$.

Esto me permite ahora volver sobre lo que expuse al final de la sección anterior, cuando presenté las intuiciones pre-teóricas como capacidades. Se dice a veces que el usuario promedio del lenguaje natural "usa incorrectamente" sus funciones lógicas cuando, por ejemplo, no entiende (ni da a entender) una afirmación en una doble negación; como cuando dice que "no hay nadie" para decir que no hay persona

instancia. Pero aquí no estamos particularmente interesados en el problema de la revisión sino en el fenómeno de la aceptación, por lo que una discusión sobre estos puntos me parece más bien marginal. 
alguna. Pero la involución de la negación (esto es, que dos negaciones "se anulen" en una afirmación) no es una propiedad intrínseca de las negaciones del lenguaje natural, sino una capacidad de estas: ellas pueden ser usadas de esta forma, y también de otras. De ahí la riqueza conceptual de la distinción entre determinable y determinantes.

Para terminar: ¿es esta artificialidad una concesión a la arbitrariedad de la Lógica? Después de todo, si la verdad de las leyes lógicas emana de la MN-L y las MN-L son artificiales, entonces todas las teorías lógicas son, o pueden llegar a ser, verdaderas. La respuesta es que sí, pero no más que cualquier otra teoría científica: "Después de todo, cualquier cosa puede causar cualquier otra. En efecto, me parece que no es implausible pensar que, con el tipo correcto de $\mathrm{MN}$, casi cualquier cosa podría llegar a necesitar cualquier otra." (Cartwright, 1999, p. 72). De igual forma, con un simbolismo adecuado pueden fabricarse los más diversos sistemas lógicos; y, con la imaginación suficiente, todos ellos podrían dotarse de una MN-L. Pero, en la práctica, esto no se da: hay sistemas lógicos que no poseen (todavía) una apropiada MN-L; y entre los que la poseen, no todos son usados en la práctica como estándar de razonamiento.

\section{La dignidad de la Lógica Clásica}

Cerca del final de la sección 2 dije que Cartwright, al introducir el concepto de MN, no está postulando entidades extrañas en la empresa científica, sino que ofrece una manera nueva de mirar lo que ya hay en ella. El concepto de MN-L debe entenderse de la misma forma: los elementos son los mismos, y en alguna medida puede decirse que la MN-L se corresponde, más o menos directamente, con lo que algunos autores han llamado la interpretación filosófica de un sistema lógico (véase, por ejemplo, Barrio y Da Ré (2018)). En este mismo sentido, me atrevería a decir que lo que distingue entre un sistema lógico puramente formal y una teoría lógica con contenido científico (i. e. interpretación filosófica) es precisamente la existencia de la MN-L ${ }^{17}$. La diferencia significativa radica en el alcance que las leyes tienen, cuando se las mira a través del prisma de Cartwright. A la luz de esta observación, en esta sección voy a volver sobre el problema que enuncié en la Introducción: el de la dignidad de la Lógica Clásica.

La Lógica Clásica es actualmente el sistema lógico aceptado como correcto. Pero ¿qué significa esto exactamente? Comencemos diciendo lo que no significa: que la Lógica Clásica capture verdades universales o necesarias acerca del mundo. No hay hechos en el mundo o en la estructura última de la realidad que se correspondan con ninguna de las leyes lógicas clásicas. (Ya lo dijera Wittgenstein también: "Una proposición de la lógica no sólo no puede ser refutada por experiencia posible alguna, sino que tampoco debe poder ser confirmada por ella" (pr. 6.1222); "El distintivo de la proposición lógica no es la validez general. Porque ser general quiere decir sólo: valer casualmente para todas las cosas" (pr. 6.1231). Esto está en la naturaleza misma de las tautologías: no sólo que son siempre verdaderas, sino que lo son por virtud de su forma.)

Una de las cosas que sí queremos decir cuando llamamos correcta a la Lógica Clásica, es que su clausura inferencial nos da todas y sólo las consecuencias que esperaríamos obtener de nuestras mejores

\footnotetext{
${ }^{17}$ Existencia de facto y no simple posibilidad, pues, como dije al final de la sección anterior, todo formalismo es en principio susceptible de ser dotado de una MN-L. Esta manera de distinguir entre "meros" formalismos y teorías lógicas qua científicas se alinea de forma bastante natural con las opiniones de Barrio y Da Ré (2018) y Hjortland (2019) sobre este mismo punto.
} 
teorías científicas. Pero esto, en sí mismo, no es suficiente: después de todo, no sabemos cuáles son esas consecuencias $\sin$ una clausura inferencial, y si las conociéramos de antemano, entonces la clausura inferencial sería innecesaria. Es necesario ahondar un poco más en este asunto.

A veces la clasicidad de la clausura se justifica apelando a propiedades formales de dicha clausura. Por ejemplo, puede demostrarse que la Lógica proposicional Clásica es completa en sentido estrecho: si le agregáramos cualquier fórmula no derivable como nuevo axioma, la haríamos explotar (Post, 1921). Esto significa que para cualquier conjunto de proposiciones no-lógicas $T$, su clausura clásica $T_{C L}$ nos da el máximo posible de consecuencias de $T$. Por lo tanto, alguien podría sostener que $T_{C L}$ nos da todo lo que necesitamos. La Lógica Clásica es la máxima teoría analítica que preserva verdad.

Sin embargo, resultados como el anterior siguen siendo altamente dependientes de que ciertas condiciones ceteris paribus se cumplan. Por ejemplo: que las proposiciones sean verdaderas o falsas de manera excluyente y exhaustiva, o que tenga siquiera sentido dar cuenta de una teoría científica en términos de conjuntos de proposiciones. Esto va sobre la pista de la misma observación que hace Cartwright sobre la dignidad de las teorías físicas para el fundamentalismo: de la decisión (filosófica) de suponer que el mundo es una gran MN se sigue que la naturaleza está gobernada por leyes naturales, y no al contrario.

El mismo alcance puede hacerse en el caso que nos ocupa: las teorías científicas mismas son fabricadas a guisa de MN-L para la Lógica Clásica: antes de aplicar la clausura, es $T$ la que ha sido diseñada para que la Lógica Clásica funcione eficientemente en ella. Dicho de otro modo, es de la decisión (filosófica) de suponer que las teorías deben ser clásicas que se sigue que la Lógica Clásica es la clausura inferencial óptima para estas teorías, y no al contrario. Y esta decisión está fundada, a su vez, en la creencia (o esperanza) de que la Razón es una gran MN-L de la Lógica Clásica - si se me permite el efectismo.

Mi uso de la palabra "decisión" en los dos párrafos anteriores podría prestarse a malentendidos. Lo que no estoy queriendo decir es que estos presupuestos filosóficos sean asumidos de manera consciente para ajustarse a un programa o ideología (no hay tal cosa como una Conspiración Clásica), o hayan sido impuestos por coerción. Pero sí creo que la adopción de este estándar y no de otro se fue dando de forma progresiva a lo largo de la historia, y que esta génesis se explica por factores sociohistóricos antes que racionales y apriorísticos.

A fin de ilustrar este último punto, permítaseme aventurar el siguiente experimento mental. Es posible imaginar un mundo en el cual no fueron los lógicos clásicos, sino los Intuicionistas, quienes ganaron el debate a principios del siglo XX sobre qué sistema lógico reconstruye mejor los razonamientos matemáticos. Hay, sin duda, una parte de esta discusión que habría involucrado razones strictu sensu, es decir, argumentos matemáticos y filosóficos. Por ejemplo: en lo que concierne a la naturaleza del infinito. Pero también otros factores históricos y socioculturales tendrían que haber jugado cierto papel: la historia del mundo tendría que haberse desenvuelto de manera ligeramente distinta a como se desenvolvió. Aunque al final, si las más grandes personalidades científicas y matemáticas del siglo pasado se hubieran formado en la escuela de Brouwer y Heyting, no es implausible que su Lógica hubiera terminado reconocida como la 'estándar'. 
Como es sabido, la Lógica Intuicionista es la Lógica Clásica menos algunas $\operatorname{cosas}^{18}$. Entre ellas están las disyunciones no analíticamente decidibles: si una disyunción tiene prueba intuicionística, entonces necesariamente uno de sus disyuntos también la tiene. Este resultado se conoce en la literatura como Propiedad Disyuntiva (PD). La ciencia de principios de siglo XXI en este mundo hipotético se habría desarrollado teniendo en mente este resultado, de manera que para sus científicos aceptar de manera irrestricta disyunciones del tipo

$$
A \vee \neg A
$$

estaría fuera de lugar; y esta sensación de extralimitación estaría justificada por una serie de creencias filosóficas acerca de qué significa que una proposición forme parte de una teoría científica, qué es la verdad (o la prueba) de una proposición, y así sucesivamente. Todas estas creencias filosóficas, más o menos explicitadas en el discurso de las científicas y filósofas de las ciencias de este mundo hipotético, provendrían de un cuentito acerca de por qué la Lógica Intuicionista es correcta ${ }^{19}$.

Ahora bien, si en este mundo alternativo la Filosofía de la Lógica llegara a plantearse la pregunta por la revisión de la Lógica Intuicionista, es claro que sus especialistas se toparían con dificultades similares a las que se encuentran en nuestro mundo las filósofas y filósofos que pretenden revisar la Lógica Clásica: una resistencia fuertemente arraigada en la tradición, en preconcepciones filosóficas implícitas y metafísicas heredadas, pero respaldada por éxitos innegables en todas las áreas donde la ciencia se haya aplicado y toda una batería de resultados metateóricos y matemáticos para dar consistencia a sus argumentos. Por ejemplo, algunas filósofas de la Lógica de este mundo ficticio podrían defender la Lógica Clásica (que forzosamente se llamaría de otra forma) como alternativa viable, y quizás adelantarían el teorema de completitud de Post como una razón. Pero las defensoras de la Lógica estándar recibirían, de seguro, este resultado como excesivo: "se ganan esquemas", dirían, "pero se pierde la PD".

Lo que quiero ilustrar con este experimento mental es que la dignidad de la Lógica Clásica no proviene exclusivamente de sus méritos formales; hay factores sociales y políticos que son igual de relevantes. Siendo justos con Cartwright, esta conclusión no es una consecuencia de la idea de MN; ni siquiera, de la de MN-L que desarrollé en las secciones anteriores. Pero me parece que la concepción particularista de las leyes lógicas, que sí es una consecuencia de aplicar el marco de Cartwright, hace más plausible la afirmación de que la aceptación generalizada de un estándar respondió a factores no necesariamente

${ }^{18}$ El hecho de que la lógica intuicionista sea sub-clásica no es trivial, porque muestra que la evidencia empírica no está jugando un papel significativo en la decisión por una u otra teoría. Dicho en otros términos: ninguna Ley de alguna de estas dos lógicas tiene contraejemplos en la otra, lo que significa que, en principio, ambas son estrictamente compatibles con toda la evidencia empírica disponible. Lo que sucederá en ocasiones es que la lógica más fuerte (en este caso, la Clásica) dirá: la ocurrencia de tales-y-tales hechos es una necesidad lógica, mientras que la lógica más débil dirá que no. Agradezco a un revisor anónimo por hacerme ver la necesidad de aclarar este punto.

19 De forma análoga a como el TLP explicita una MN-L para la Lógica Clásica, hay textos en la literatura sobre intuicionismo que explicitan su MN-L. Por ejemplo: Brouwer (1996; 1948). Véase Placek (1999) para una exposición crítica de las bases filosóficas del intuicionismo. 
apriorísticos, pues debilita la caricatura kantiana del Agente Racional inspeccionándose a sí mismo y asintiendo a las leyes que está obligado a aceptar por su propia naturaleza.

Volvamos ahora la mirada sobre los programas abductivistas en Filosofía de la Lógica. Se dice que, en concordancia con el A-EL, las teorías lógicas deberían ser sensibles a la evidencia, y su elección debería responder a criterios racionales de adecuación a dicha evidencia: inferencia a la mejor explicación, contrastación de virtudes epistémicas, etc. (véase Hjortland (2017; 2019)). Se ha dicho en ocasiones (Finn, 2019) que el abductivismo está viciado en el caso de la Lógica, porque, a diferencia de las demás ciencias, en el caso de la Lógica necesitas asumir la lógica misma para justificar su revisión; mi opinión es que la situación es todavía más compleja. No es sólo que el abductivismo en ciencias se base en la Lógica Clásica: son las teorías en sí mismas las que han sido concebidas y diseñadas para responder a la Lógica Clásica ${ }^{20}$. Por lo tanto, parte de lo que sería necesario para dar lugar a una revisión sería permitir que los alcances de otras lógicas fueran explorados.

Mi conclusión en este punto es, pues, agridulce. Por una parte, me parece que la Lógica Clásica sí puede revisarse, pues su dignidad ni se funda enteramente en ella misma, ni se funda en la naturaleza última de la razón o de lo real; esto sería lo dulce. Lo agrio viene al notar que esta revisión pasa por un proceso mucho más arduo que el asumido habitualmente por las abductivistas. Erigir una nueva lógica implica fabricar nuevas MN-L, pero también defenderlas y eventualmente popularizarlas. Las dos primeras pueden parecerse a lo que las abductivistas entienden por revisar la Lógica Clásica; pero lo tercero se da en un proceso sociocultural e histórico, y es sabido que estos últimos muy pocas veces pueden ser dirigidos o predichos racionalmente.

\section{Conclusiones}

Las anti-excepcionalistas lógicas creen que la Lógica es como cualquier otra ciencia. Si esta afirmación es cierta, entonces no sólo ella es revisable como toda teoría científica, sino que además todo lo que se puede decir sobre las ciencias aplica, mutatis mutandis, también para ella. El propósito de este artículo ha sido explorar esta consecuencia del A-EL, acercando el marco conceptual de Cartwright (1999) a la Filosofía de la Lógica.

Cartwright afirma que las leyes científicas no capturan leyes naturales, porque, para ella, la naturaleza no está gobernada por leyes. Lo que hay de verdadero en las teorías que la Ciencia formula no está en el mundo sino en los modelos de dichas teorías: sistemas complejos, altamente controlados, suficientemente estables, artificiales y específicos. Yo he mostrado que lo mismo puede decirse de la Lógica: ella no captura las Leyes del Pensamiento o de la Razón, porque el pensamiento y la razón no están gobernados necesariamente por leyes. En cambio, sobre lo que sí puede decirse que versan las Leyes de la Lógica son metafísicas posibles: historias filosóficamente simples, pero comprometidas, acerca de qué son las proposiciones, el pensamiento, la verdad y la validez.

\footnotetext{
${ }^{20}$ Se dirá: "con la Lógica Clásica pudimos llegar a la luna”. Concedamos por el momento que esto cuente como evidencia en favor de la Lógica (cosa que no creo). ¿Qué industria, qué ingeniería, qué física ha intentado hacer cohetes asumiendo otra Lógica como clausura inferencial de sus teorías? Es fácil ganar una carrera cuando se es la única participante.
} 
Esta imagen de la Lógica impacta en el problema de la revisión de la Lógica Clásica, en la medida en que muestra que dicha revisión no necesariamente responde a un proceso racional de adecuación de la teoría a los hechos, porque los "hechos" relevantes no son anteriores a la teoría misma, sino que, en alguna medida, están moldeados y conducidos por ella.

\section{Referencias}

Barrio E. y Da Ré, B. (2018). "Paraconsistency and its philosophical interpretations." The Australasian Journal of Logic, 15(2): 151-170. doi: https://doi.org/gm45

Barros Grez, D. (1888). Fábulas Orijinales. Memoria Chilena. Biblioteca Nacional de Chile. Consulta 20 abril de 2021. URL: http://www.memoriachilena.gob.cl/602/w3-article-9691.html

Beall, J.C., Restall, G., Sagi, G. (Spring 2019 Edition). Logical Consequence. The Stanford Encyclopedia of Philosophy. Edward N. Zalta (ed.) URL: https://plato.stanford.edu/archives/spr2019/entries/logical-consequence/

Brouwer, L. E. J. (1996). “Life, Art, and Mysticism” Notre Dame Journal of Formal Logic, 37 (3): 389 - 429. doi: https://doi.org/ckkjrg

Brouwer, L. E. J. (1948). “Consciousness, Philosophy and Mathematics”. En A. Heyting (ed.) Collected works of L. E. J. Brouwer I: Philosophy and Foundations of Mathematics (480-494). Amsterdam: North-Holland Publishing.

Cartwright, N. (1980). "The truth doesn't explain much.” American Philosophical Quarterly, 17(2): 159-163. doi: https://doi.org/fqzdjt

Cartwright, N. (1999). The dappled world: A study of the boundaries of science. Cambridge University Press. doi: https://doi.org/gm46

Da Costa, N. (1980). Ensaio sobre os fundamentos da lógica. Sao Paulo: Hucitec Edusp.

Dennett, D. (1995). “Intuition Pumps”. En J. Brockman (ed.), The Third Culture (181-197). New York et al.: Simon \& Schuster.

Dennett, D. (2013). Bombas de intuición y otras herramientas de pensamiento. Traducción de Laura Lecuona. México D. F.: Fondo de Cultura Económica.

Finn, S. (2019). "The adoption problem and anti-exceptionalism about logic.” The Australasian Journal of Logic [S.I.], 16(7): 231-249. doi: https://doi.org/gm47

Hjortland, O. (2017). “Anti-Exceptionalism about logic.” Philosophical Studies, 174: 631-658. doi: https://doi.org/ghmszs

Hjortland, O. (2019). "What counts as evidence for a logical theory?" The Australasian Journal of Logic, 16(7): 250-282. doi: https://doi.org/gm48

King, P., Arlig, A. (Fall 2018 Edition) Peter Abelard. The Stanford Encyclopedia of Philosophy, Edward N. Zalta (ed.). URL: https://plato.stanford.edu/archives/fall2018/entries/abelard/ 
Placek, T. (1999). Mathematical intuitionism and intersubjectivity. A critical exposition of arguments for intuitionism. Dordrecht: Springer. doi : https://doi.org/gm49

Popper, K. R. (1965). “Indeterminismo y voluntad humana.” En David Miller (ed), (1995) Popper: Escritos selectos. Traducción de Sergio Madero Báez. Ciudad de México: Fondo de Cultura Económica.

Post, E. (1921). "Introduction to a general theory of elementary propositions." American journal of mathematics, 43: 169-173. doi: https://doi.org/bxq6d4

Priest, G. (2006). In Contradiction: a Theory of the Transconsistent. Oxford: Oxford University Press. doi: https://doi.org/brb5cv

Russell, G. (Summer 2021 Edition). Logical Pluralism. The Stanford Encyclopedia of Philosophy, Edward N. Zalta (ed.). URL: https://plato.stanford.edu/archives/sum2021/entries/logicalpluralism/

Sylvan, R. (1999). "What is that item designated negation?” En Gabbay, D. y Wansing, H. (eds.) What is negation? Dordrecht: Springer. doi: https://doi.org/gm5b

Tajer, D. (2020) "Intuiciones en lógica: una propuesta moderada." Revista de Humanidades de Valparaíso, 16: 239-253. doi: https://doi.org/gm5c

Williamson, T. (2013). “What is naturalism?” En Haug, M. (ed.) Philosophical Methodology: The Armchair or the Laboratory? London; New York: Routledge.

Williamson, T. (2014). "Logic, metalogic and neutrality." Erkenntnis, 79(2): 211-231. doi: https://doi.org/gm5d

Wittgenstein, L. (2014). Investigaciones Filosóficas. Traducción y notas de Alfonso García Suárez y Carlos Ulises Moulines. Madrid: Editorial Gredos.

Wittgenstein, L. (2018). Tractatus Logico-Philosophicus. Traducción de Jacobo Muñoz e Isidoro Reguera. Madrid: Editorial Gredos. 